\author{
${ }^{1}$ Museum of Natural History Neuchâtel, 2000 \\ Neuchâtel, Switzerland, ${ }^{2}$ Department of \\ Ecology and Evolutionary Biology, University \\ of Arizona, PO Box 210088, Tucson, AZ 85721, \\ USA, ${ }^{3}$ Molecular Systematics Section, Jodrell \\ Laboratory, Royal Botanic Gardens, Kew, \\ Richmond, Surrey TW9 3DS, UK, \\ ${ }^{4}$ Department of Biodiversity and Conservation, \\ Real Jardin Botanico, CSIC, 28014 Madrid, \\ Spain, ${ }^{5}$ Unit of Ecology and Evolution, \\ Department of Biology, University of Fribourg, \\ 1700 Fribourg, Switzerland, ${ }^{6}$ Department of \\ Ecology and Evolution, University of Lausanne, \\ Biophore Building, 1015 Lausanne, \\ Switzerland
}

*Correspondence: Nadir Alvarez, Department of Ecology and Evolution, University of Lausanne, Biophore building, 1015 Lausanne, Switzerland. E-mail: nadir.alvarez@unil.ch

${ }^{\dagger}$ These authors contributed equally to this work and are considered as joint first authors.

\section{Climate oscillations and species interactions: large-scale congruence but regional differences in the phylogeographic structures of an alpine plant and its monophagous insect}

\author{
Matthias Borer $^{1 \dagger}$, Nils Arrigo ${ }^{2 \dagger}$, Sven Buerki ${ }^{3,4 \dagger}$, Russell E. Naisbit ${ }^{5}$ and \\ Nadir Alvarez ${ }^{6 *}$
}

\section{ABSTRACT}

Aim To predict the fate of alpine interactions involving specialized species, using a monophagous beetle and its host plant as a case study.

Location The Alps.

Methods We investigated genetic structuring of the herbivorous beetle Oreina gloriosa and its specific host-plant Peucedanum ostruthium. We used genome fingerprinting (in the insect and the plant) and sequence data (in the insect) to compare the distribution of the main gene pools in the two associated species and to estimate divergence time in the insect, a proxy for the temporal origin of the interaction. We quantified the similarity in spatial genetic structures by performing a Procrustes analysis, a tool from shape theory. Finally, we simulated recolonization of an empty space analogous to the deglaciated Alps just after ice retreat by two lineages from two species showing unbalanced dependence, to examine how timing of the recolonization process, as well as dispersal capacities of associated species, could explain the observed pattern.

Results Contrasting with expectations based on their asymmetrical dependence, patterns in the beetle and plant were congruent at a large scale. Exceptions occurred at a regional scale in areas of admixture, matching known suture zones in Alpine plants. Simulations using a lattice-based model suggested these empirical patterns arose during or soon after recolonization, long after the estimated origin of the interaction $c .0 .5$ million years ago.

Main conclusions Species-specific interactions are scarce in alpine habitats because glacial cycles have limited the opportunities for co-evolution. Their fate, however, remains uncertain under climate change. Here we show that whereas most dispersal routes are paralleled at a large scale, regional incongruence implies that the destinies of the species might differ under changing climate. This may be a consequence of the host dependence of the beetle, which locally limits the establishment of dispersing insects.

\section{Keywords}

AFLP, congruence testing, Europe, Oreina gloriosa, Peucedanum ostruthium, phylogeography, Procrustean analyses, Quaternary, random walk model, spatial genetic structure. 


\section{INTRODUCTION}

Species-specific ecological interactions are scarce in alpine habitats because the ebb and flow of Quaternary glaciations has limited the time-scale over which long-term co-evolutionary processes occur due to recurrent local extinctions and continually moving species ranges (Rasmann et al., in press; see also Jablonski, 2008). Exceptions are found in several biological groups, notably plants and phytophagous insects (Dobler et al., 1996). However, the fate of such interactions remains uncertain under scenarios of climate change, and species involved in the interaction might be threatened unless they have evolved strategies for co-dispersing in order to follow their abiotic and biotic ecological clines (Alvarez et al., 2009). A proxy for evaluating whether species demonstrating highly specific interactions may be able to track climate change is to examine their phylogeographic patterns, and thus the level of spatial congruence in their dispersal routes and refugia occupied during Quaternary climatic oscillations (Emerson \& Hewitt, 2005).

Pleistocene climatic oscillations strongly influenced the genetic structure of organisms in the Palaearctic (Bennett, 1990; Hewitt, 1996). This has been best studied for the glacial refugia and recolonization patterns of lowland temperate species, and several paradigms have been identified (Taberlet et al., 1998; Hewitt, 2000). For instance, Mediterranean insects have been shown to occupy latitudes as high as the British Isles during the Eemian interglacial (Coope, 1990). When the climate cooled during the last glacial cycle, their ranges retracted to the southern edge of the European continent and they subsequently recolonized northern territories during the current interglacial (Schmitt, 2007). In contrast, such effects are less well documented in cold-adapted taxa such as those with current alpine distributions. In the past, these species would have been much more broadly distributed than they are today, especially during the ice age maxima when some of them occupied the extensive European steppe (see reviews in Willis \& van Andel, 2004; Stewart et al., 2010). Not all coldadapted species experienced similar changes in their genetic structure; for instance, the genetic diversity of alpine plants has been shown to depend on specific life-history traits (e.g. Thiel-Egenter et al., 2009). From such evidence it can be expected that climatic oscillations not only influenced the evolutionary histories of single species, but also ecological interactions (Jablonski \& Sepkoski, 1996; Mouritsen \& Poulin, 2002): from one glacial cycle to another, the species composition of alpine communities would have differed according to the ability of populations to recolonize suitable habitats, but also due to stochastic processes in population dynamics (Jackson \& Overpeck, 2000). Hence, the evolution of specific interactions among European taxa might be rearranged at each glacial cycle, with emerging co-evolutionary processes being abruptly initiated or halted. To date, the few studies investigating phylogeographic patterns in interacting species have been focused on temperate organisms (Hayward \& Stone, 2006; Bruyndonckx et al., 2009; Espíndola \& Alvarez,
2011) and underlying processes have rarely been addressed. It is thus timely to investigate this question in cold-adapted organisms.

Here we focus on an antagonistic interaction involving an Alpine leaf beetle, Oreina gloriosa (Fabricius) (Coleoptera: Chrysomelidae), and its sole host plant, masterwort, Peucedanum ostruthium (L.) W. D. Koch (Apiaceae), using genetic data and random walk models. The plant is trophically and reproductively independent from the insect and is protected against generalist herbivores by coumarins (Hadaček et al., 1994), a chemical defence strategy that the specialist O. gloriosa is able to circumvent. Hence two competing hypotheses are proposed. On the one hand, the evolutionary histories of the two interacting species might be incongruent, with colonization of the landscape by the host plant occurring independently of the spread of its dependent species (i.e. the insect switches frequently among host plant lineages). This hypothesis also includes escape situations between plant and insect lineages, for instance when the former colonizes regions not yet accessible to the latter. On the other hand, congruent evolutionary histories could be anticipated if the dependent organism is strongly constrained in its migration pathways. Here, the two species disperse in parallel, with the insect following the routes of the plant, resulting in congruent phylogeographic scenarios within the range of the beetle: although differences in the species' life-history traits (e.g. population sizes, generation times) might blur these simple predictions (Alvarez et al., 2010), the results will inform us as to the capacity of this interaction to be maintained in the face of climatic oscillations.

Our aim was to test these hypotheses by first comparing the spatial genetic structures of O. gloriosa and its host plant $P$. ostruthium using amplified fragment length polymorphism (AFLP) genome fingerprinting across the entire geographic distribution of the beetle (i.e. the Alps). After evaluating the respective spatial genetic structures of the two species we assessed their level of congruence by using Procrustes analysis, a statistical procedure newly introduced in phylogeography (Larmuseau et al., 2010). This statistical tool, derived from the shape theory family (Kendall, 1989), has been widely applied in morphometrics (Klingenberg \& McIntyre, 1998) and ecology (Jackson, 1995). It allows the comparison of several datasets describing the same set of points (here, the sites where plants and insects were collected). As it addresses both global and local congruence patterns among these points, it is a suitable tool for comparing the phylogeography of organisms with overlapping ranges. This tool has been shown to be potentially more powerful and flexible than Mantel tests (Peres-Neto \& Jackson, 2001). This approach was complemented by a molecular phylogeny based on sequencing of three mitochondrial regions in O. gloriosa, dated using average substitution rates in insect mitochondrial DNA (mtDNA), in order to determine the time frame of divergence in the co-evolutionary histories of the two species. Finally, we investigated the role of life-history traits, such as dispersal capacity, on the congruence of phylogeographic 
patterns, by implementing a lattice-based random-walk demographic model.

\section{MATERIALS AND METHODS}

\section{Biological material and sampling}

The host plant, P. ostruthium, is a widespread subalpine to alpine, perennial herbaceous plant occurring in most European mountains, including the Massif Central, the Pyrenees, Corsica, the northern Apennines and the Alps (Aeschimann et al., 2004). The beetle species, O. gloriosa, is found in isolated populations, with a distribution restricted to the Alps (Kippenberg, 1994). Like most Oreina species it possesses warning coloration and chemical defence; it is one of the few species that are highly specialized on a single host plant (Dobler et al., 1996), although it has not spread throughout the entire distribution of its host.

Between 2004 and 2008, O. gloriosa specimens were collected from 22 populations (one to three beetles per site) covering the whole Alpine distribution and following the grid established for the IntraBioDiv mapping of the Alpine and Carpathian flora (see Appendix S1 in Supporting Information; see also further details in Gugerli et al., 2008). Specimens were preserved in pure ethanol and stored at $-20{ }^{\circ} \mathrm{C}$. Only males were included in genetic analyses in order to allow accurate species identification based on genitalia (Kippenberg, 1994). Sampling was completed with two outgroup specimens selected from the most closely related beetle species, Oreina speciosa (Hsiao \& Pasteels, 1999). Total genomic DNA was extracted from four legs of each Oreina specimen using a DNeasy Blood \& Tissue Kit (Qiagen, Hilden, Germany) (see Borer et al., 2010, for more details).

For the host plant P. ostruthium, phylogeographic patterns were retrieved from data provided by the IntraBioDiv consortium (Gugerli et al., 2008; Alvarez et al., 2009).

\section{The AFLP dataset}

AFLP analyses were performed following Vos et al. (1995). For the beetle, three primer pairs were used (EcoRI-ACA/ MseI-AGC, EcoRI-ACA/MseI-ACG and EcoRI-ACA/MseI$\mathrm{AAC})$ and reactions were conducted using 96-well plates in which samples were randomly distributed. Fifteen individuals were amplified twice in order to determine the overall reproducibility rate. Peak Scanner 1.0 (Applied Biosystems, Foster City, CA, USA) was used to detect and measure the size of AFLP bands along electropherograms (Applied Biosystems; using default peak detection parameters with the addition of a light peak smoothing). The scoring (i.e. recording presence/absence of AFLP bands for each specimen) was performed using RAwGeno 2.0, an automated scoring R package (Arrigo et al., 2009). The analysis was set as follows: scoring range, 100-300 bp; minimum intensity, 50 relative fluorescence units (rfu); maximum bin width, $1 \mathrm{bp}$; and removal of closely sized bins (5\%). Finally, results from the three primer pairs were pooled into binary matrices before being further processed.

\section{Bayesian clustering of AFLPs}

The spatial genetic structure of the beetle was investigated using model-based Bayesian clustering of AFLPs, as implemented in structure 2.2 (Falush et al., 2007). Specimens were assigned into a number of genetic groups defined by the user (hereafter $K$ ), defined in such a way as to maximize the within-group Hardy-Weinberg equilibrium. We used the 'admixture' model (i.e. default settings) and set the Markov chain Monte Carlo (MCMC) with 100,000 generations for burn-in and 400,000 generations for data acquisition. The number of groups was screened for values ranging between one and nine (five replicates per $K$-value). The selection of the most likely $K$-value was achieved following Evanno et al. (2005) and only the run obtaining the highest likelihood in the optimal $\mathrm{K}$-value was taken into account. Handling of struCTURE results was achieved using SIMIL, an R script collection (Alvarez et al., 2008). Specimens were assigned to a given STRUCTURE group when their assignment probability exceeded 0.5 (i.e. the majority-rule criterion).

For the spatial genetic structure of the plant, we used STRUCTURE results published in Alvarez et al. (2009). The analysis used the no-admixture model and uncorrelated allele frequencies. The MCMC chain was set with 200,000 burn-in generations followed by $1,000,000$ generations of data acquisition.

Results for the plant and the insect were displayed on geographical maps using ArcGIS 9.1 (ESRI, Redlands, CA, USA), with populations being displayed as pie-charts showing the number of individuals assigned to each STRUCTURE group.

Finally, the global congruence between the phylogeographic patterns of beetles and plants was assessed by applying Mantel tests between distance matrices (Jaccard index, 100,000 permutations; Legendre \& Lapointe, 2004) computed from AFLP datasets of both species. Fine-scale congruence patterns were investigated using a Procrustes rotation analysis (Kendall, 1989; as implemented in the ADE-4 R package; Thioulouse et al., 1996). The Procrustes rotation is primarily a shape analysis for comparing two distinct datasets (here the STRUCTURE assignment probabilities for the plant and the beetle, averaged at the population level) describing the same objects (here the sites where specimens were collected). The analysis works as follows: (1) the beetle and plant datasets are reduced to two-dimensional spaces, using principal components analysis and retaining the two most significant eigenaxes, (2) new coordinates are computed for both datasets in such a way as to optimize their global matching, by adjusting translational, rotational and scaling components, and (3) because these adjustments apply globally, one can measure by computing Euclidean similarities between the adjusted datasets how congruence is distributed spatially (i.e. at the level of each plant and beetle population). To cross-check the results with a non-model based approach, the complete Procrustes 
procedure was repeated using the population differentiation statistic (i.e. $G_{\mathrm{ST}}$ values computed from the AFLP datasets, based on the Shannon diversity index) as a starting dataset, instead of STRUCTURE-based probability assignments. Both approaches provided congruent patterns (correlation $=0.51$, $P$-value $=0.02$; see Appendix S2) and only results obtained with Structure were mapped using ArcGIS 9.1.

\section{Sequencing and alignment}

Three mtDNA regions were amplified in beetles using universal insect primers: partial $16 S$ ribosomal RNA (hereafter 16S) (modLR-J-12887 with LR-N-13398; Simon et al., 1994); cytochrome $c$ oxidase subunit I (COI) (C1-J-1751 with C1-N2191; Simon et al., 1994); and cytochrome $c$ oxidase subunit II (COII) (modTL2-J-3037 with modC2-N-3661; Mardulyn et al., 1997). Polymerase chain reaction amplifications were performed using a standard protocol (see Borer et al., 2010). Sequencing was carried out by Macrogen Inc. (Seoul, South Korea) with both forward and reverse primers using an ABI 3730XL DNA Analyzer automated sequencer (Applied Biosystems, Foster City, CA, USA). Sequences (HQ 646406-HQ 646551) were manually corrected using the software CHroMasPro 1.34 (Technelysium Ltd, Helensvale, Qld, Australia). The protein-coding nucleotide sequences of $\mathrm{COI}$ and COII were checked for reading frame errors and termination codons in MEgA4 (Tamura et al., 2007). Alignments were carried out using ClustalW (Thompson et al., 1994) within the software BioEdit 7.0.5.3 (Hall, 1999), followed by minor manual correction.

\section{Phylogenetic analyses and divergence time estimation}

A partitioned Bayesian analysis was performed using BEAST 1.4.7 (Drummond \& Rambaut, 2007) with the three mtDNA regions represented as separate partitions (with specific rates of substitution; see below). Best-fit models - HKY for $16 \mathrm{~S}$ and $\mathrm{HKY}+\mathrm{G}$ for COI and COII, respectively - were estimated for each partition using MrModeltest 2.3 (Nylander, 2004). Three independent runs of $30 \times 10^{6}$ generations were performed, sampling one tree every 1000 generations. For each parameter, convergence of the independent runs was confirmed by the examination of their respective distributions in Tracer 1.4 (Rambaut \& Drummond, 2007). After removing a burn-in period of $10 \times 10^{6}$ generations in each run, $95 \%$ divergence time interval confidences were plotted on a majority rule consensus tree (reconstructed using PAUP* 4.0b10; Swofford, 2003) using TreeAnnotator (Drummond \& Rambaut, 2007). The topology was checked using the parsimony ratchet approach following Buerki et al. (2009). Node support was determined by computing Bremer support values as implemented in TreeRot 3 (Sorenson \& Franzosa, 2007) and using PAUP $^{*} 4.0 \mathrm{~b} 10$ with maxtrees $=1000$ and nreps $=10$. Fossil records are not available to calibrate estimates of Oreina divergence time, so our analysis relied on gene-specific mtDNA substitution rates from diverse arthro- pod taxa (recently reviewed in Borer et al., 2010). Average pairwise divergence substitution rates were $0.61,1.73$ and $1.38 \%$ per million years for $16 S, C O I$ and COII, respectively. Based on these values, a relaxed clock with log-normal branch length distribution was performed following Borer et al. (2010).

\section{Random-walk modelling}

We simulated plant and beetle populations in order to model the evolution through time of phylogeographic patterns that could be expected in this unbalanced interaction (i.e. the insect alone is dependent on the plant) under a simple random dispersal model. Simulations were performed under the $\mathrm{R}$ environment (script available on request from the authors) on a 25 cell $\times 25$ cell grid, with each grid cell potentially sheltering a population of 20 plants and 20 insects. Simulations were initiated with an empty grid, with landscape colonization starting from two refugium cells located at opposite edges of the grid. Plants and insects shared the same refugia (starting positions located at $x_{\text {refugium } 1}=0, y_{\text {refugium } 1}=13$ and $x_{\text {refugium2 }}$ $=25, y_{\text {refugium } 2}=13$ ). Plants could spread to and survive in any grid cell. In contrast, insects could establish only where plants were already present.

Each generation of the simulation process included: (1) a population growth phase, (2) a dispersal phase, and (3) an extinction phase. The population growth phase increased the number of individuals in each cell by $50 \%$ (i.e. one offspring was added to the population for each pair of individuals present in the current generation). The dispersal phase relied on: (1) the number of dispersal events per cell, determined using a binomial distribution (with migration probability $M$ being 5 or $10 \%$ for each individual in the cell; see below), (2) the number of migrants per dispersal event, following a Poisson distribution (with $\lambda=2$ ), and (3) the distance reachable by each migrant individual (Poisson distribution, $\lambda_{\mathrm{d}}=1$ or 2 cell units; see below). The direction of migration was determined randomly. The distributions were set empirically to produce either a slow or a rapid dispersal speed ( $M=5 \%$ and $\lambda_{\mathrm{d}}=1$ for slow dispersal; $M=10 \%$ and $\lambda_{\mathrm{d}}=2$ for rapid dispersal). Finally, the extinction phase removed individuals from each cell, using a binomial distribution (with a $5 \%$ probability of death for each individual).

Four scenarios were tested by combining the respective dispersal speeds of plants and insects: 'slow plant and slow insect', 'slow plant and rapid insect', 'rapid plant and slow insect' and 'rapid plant and rapid insect'. For each scenario, the set-up timing was defined as the point at which $90 \%$ of all cells were colonized by both the plant and the insect. The scenarios differed in their set-up timing, as $124 \pm 5$ generations were needed for the scenario 'slow plant and slow insect', $114 \pm 6$ for 'slow plant and rapid insect', $107 \pm 6$ for 'rapid plant and slow insect' and $64 \pm 2$ for 'rapid plant and rapid insect'. Phylogeographic data were collected at the 25th and 100th generations after the set-up timing (i.e. hereafter gen $=25$ and $g e n=100$, respectively) . 
All simulations were replicated 50 times to produce averaged measures and the Procrustes rotation approach described above was applied to assess congruence between plant and insect phylogeographic patterns. Finally, results were displayed as population growth curves and maps.

\section{RESULTS}

\section{AFLP analyses and spatial genetic structures}

In O. gloriosa, the AFLP analysis produced a total of 497 bands (154, 156 and 187 for EcoRI-ACA/MseI-AGC, EcoRI-ACA/ MseI-ACG and EcoRI-ACA/MseI-AAC, respectively) with an average of 257 bands per individual and $89 \%$ overall signal reproducibility in the 15 resampled individuals. The spatial genetic structure of the beetle was investigated using modelbased Bayesian clustering methods implemented in STRUCTURE 2.2. The most likely results (i.e. runs maximizing the likelihood estimate) were obtained when considering six groups $(K=6)$. Populations were mostly composed of specimens assigned to a single group, with the exception of three Swiss populations comprising beetles from two genetic lineages (CDM, GLT and SAG) (Fig. 1a). Populations from the eastern Alps were assigned to a single cluster (shown in purple), with the exception of the VDD population from the Trentino area, assigned to the same group as most populations from the central Alps (in yellow). Populations from the Alpes-Maritimes were assigned to a spatially restricted cluster (in red), while the western Alps mostly comprised individuals from one single widespread cluster (in green). Finally, admixed individuals (i.e. not attributed to any cluster using the majority-rule criterion) were observed in three populations from the central Alps (SAG, GON and LDT) (Fig. 1a).

In $P$. ostruthium, the most likely $K$-value in STRUCTURE runs was obtained with five groups spread across the Alps (see Alvarez et al., 2009) (Fig. 1b). The eastern Alps were split into a northern and a southern group (in purple and blue), one group covered the southern part of Switzerland (in yellow), one the western Alps (in green) and the fifth occurred in the Maritime and western Alps (red) (Fig. 1b).

\section{Comparison between the phylogeographic patterns of $\boldsymbol{O}$. gloriosa and $\boldsymbol{P}$. ostruthium}

Overall, the plant and insect AFLP phylogeographies were congruent, as shown by a significant Mantel statistic (using Spearman's correlation, $r=0.275 ; P$-value $=0.01)$. Furthermore, the Procrustes rotation analysis allowed an accurate quantification of regional congruence among the plant and beetle phylogeographies. The obtained results ranged between 0 (i.e. total incongruence) and 1 (i.e. total congruence) and were distributed bimodally, with a marked threshold at 0.5 ,

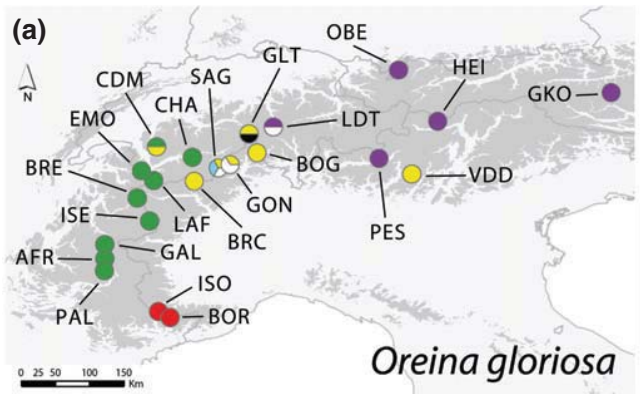

(c) $\hat{N}_{N}$

Procrustes analysis

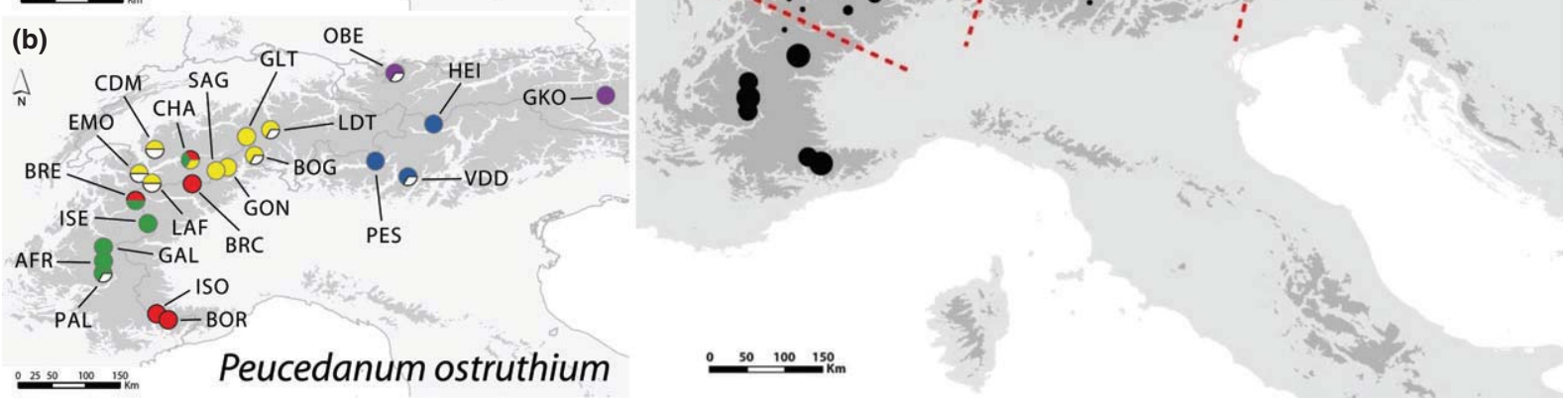

Figure 1 Spatial genetic structures of Alpine populations of Oreina gloriosa and Peucedanum ostruthium, and overall congruence, as revealed by amplified fragment length polymorphism (AFLP) analysis. Phylogeographic patterns of (a) O. gloriosa (structure Bayesian clustering, $K=6$ ) and (b) P. ostruthium (structure Bayesian clustering, $K=5$ ). Admixed individuals (i.e. with assignment probabilities lower than 0.5) were left as unassigned to genetic groups and are shown as white in the pie-charts. Each group is represented by a colour that has been selected to maximize the overlap between patterns from the two species. (c) Congruence patterns between plant and insect AFLP signals, measured using Procrustes rotation analysis. The circle diameter is proportional to the local agreement between plant and insect spatial genetic structures (see text), i.e. the larger the circle, the higher the level of congruence at the scale of a single population. Dashed lines show the major genetic suture zones of the Alpine arch, as reported in Schönswetter et al. (2005). Topographic shading in the Alps shows elevations above $1000 \mathrm{~m}$. Letters refer to sampled populations. A key to the population codes is given in Appendix S1. 
above which populations were considered as congruent for the plant and the insect (Fig. 1c). Several discrepancies between plant and beetle phylogeographies were revealed at the regional scale (Fig. 1c), with Procrustes values $<0.5$, in areas corresponding to biogeographic suture zones and within-species genetic break zones (Schönswetter et al., 2005; Thiel-Egenter et al., 2011).

\section{Phylogenetic reconstruction and divergence time estimates}

Total alignment length of the concatenated mtDNA dataset was $1626 \mathrm{bp}$ (with 529, 462 and 635 bp for 16S, COI and COII, respectively). Among 50 variable characters in the combined dataset, excluding the outgroup, 29 were potentially parsimony informative (representing $3.1 \%$ of the total). Phylogenetic reconstructions were performed using partitioned Bayesian and parsimony ratchet analyses. Both approaches supported the monophyly of O. gloriosa (Fig. 2; Appendix S3a). Within the ingroup, the two phylogenetic analyses recovered three well-supported clades with Bayesian posterior probabilities $>0.9$ and Bremer supports $>2$ in the parsimony ratchet analysis (Fig. 2a, clades I to III). Five specimens (995_BRC, 359_ISE, 406_BOR, 1132_GAL and 1133_GAL) were embedded in a polytomy closely related to clades II and III. Because the parsimony ratchet and Bayesian-based phylogenetic hypotheses were highly congruent only the latter will be discussed further (it has the benefit of also providing an estimate of the timing of divergence between lineages). Divergence time analysis estimated the origin of the three main clades as occurring before the Last Glacial Maximum (Fig. 2a), with a $95 \%$ confidence interval spanning the last

\section{(a)

(b)
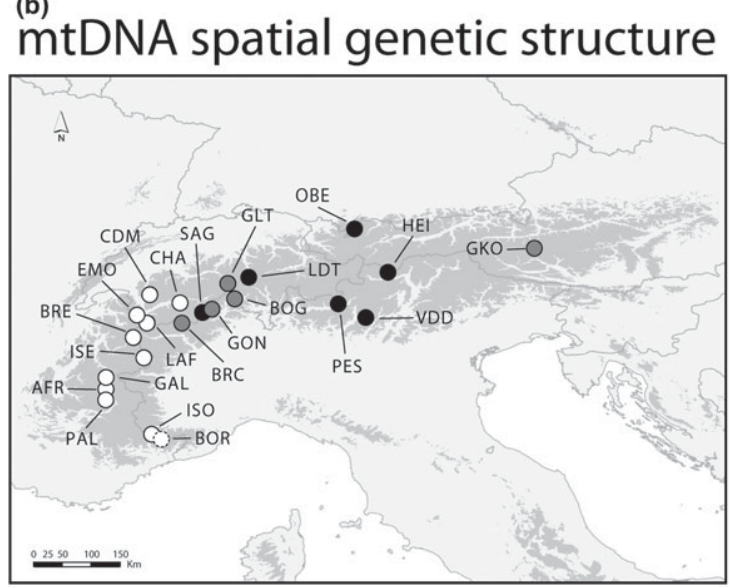

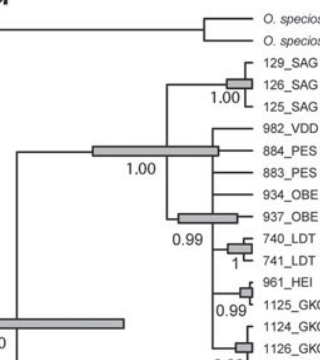

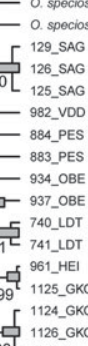
0.93 [962_HEI

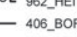

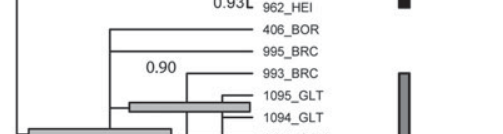

clade II

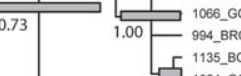

0.84 1064_GON 1 1065_GON
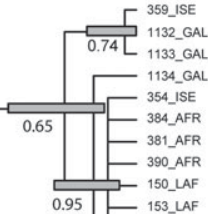

$0.95[$ - 153_LAF

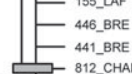

0.86 - 812 _CHAN

clade III

\begin{tabular}{c|c|c|c|cc}
\hline 1 & & & & & \\
2.0 & 1.5 & 1.0 & 0.5 & 0 & $\mathrm{Ma}$
\end{tabular}

Figure 2 (a) Majority-rule consensus tree of the alpine leaf beetle Oreina gloriosa mitochondrial DNA (mtDNA) dataset with two outgroup specimens of the closely related beetle species Oreina speciosa. The topology is computed from the Markov chain Monte Carlo stationary distribution of the partitioned coalescent Bayesian divergence time analysis, with median values and $95 \%$ confidence intervals for nodal ages. Values at the nodes are Bayesian posterior probabilities. The three main clades are indicated with vertical bars in black, grey and white. (b) Spatial distribution of the 22 beetle populations showing their clustering in the three main clades defined in (a). Topographic shading in the Alps shows elevations above $1000 \mathrm{~m}$. Letters refer to sampled populations; see Appendix S1 for a key to the population codes. Population BOR, which does not include any individual in one of the three resolved clades, is marked with a dotted circle. 
0.5 million years (Myr). Clade I included specimens from the eastern and central Alps and diverged c. 0.5 million years ago (Ma), followed quickly by the radiation of clades II and III (i.e. within $0.3 \mathrm{Myr}$, estimated as crown age for the corresponding polytomy), containing specimens from the central Alps and from the western and south-western Alps, respectively (Fig. 2b).

The mtDNA and AFLP phylogenetic patterns were highly congruent (see Appendix S3b): mtDNA clade I matched the purple STRUCTURE group at 77\%, clade II matched the yellow group at $83 \%$ and clade III matched the green group at $85 \%$. Other stRUCTURE groups comprising fewer specimens also matched with a given mtDNA clade: the light blue group with clade I, the black group with clade II and the red group with clade III.

\section{Random-walk models}

Simulations of dispersal in the plant and the beetle were performed to provide insights into the evolution of phylogeographic patterns under a simple random dispersal model. The simulations considered a lattice-based landscape where each grid cell potentially sheltered a plant and an insect population. Each population relied on a demographic model including a growth phase, a dispersal phase and an extinction phase. The simulations were initiated with an empty grid (i.e. a proxy of post-glacial deglaciated areas), with landscape colonization starting from two refugium cells located at opposite edges of the grid. Plants could spread and survive in any grid cell. In contrast, insects could establish only where plants were already present. Finally, four scenarios combining either slow or rapid dispersal abilities for the plant and the insect (Fig. 3) were considered. All simulations followed a typical scheme, starting with colonization of the landscape associated with clear-cut phylogeographic patterns (e.g. Fig. 3a; shown 25 generations after $90 \%$ of the grid was colonized, i.e. gen $=25$ ) followed by a homogenization phase with phylogeographic patterns vanishing through time (e.g. Fig. 3a; results after 100 generations, i.e. gen $=100$ ). In three out of four scenarios (Fig. 3a,b,d) Procrustes rotation analyses revealed that phylogeographic patterns of plants and beetles were globally congruent in the initial period after complete colonization of the landscape (i.e. at gen $=25$ ). The three scenarios also yielded suture zones in the middle of the grid, where lineages expanding from different refugia met. These suture zones were associated with low phylogeographic congruence between plants and insects (as highlighted by black horizontal arrows in Fig. 3a-d). Measurements made later during the homogenization phase (i.e. gen $=100)$ showed suture zones that were less clear and a decrease in phylogeographic congruence. The fastest loss of congruence was observed in the third scenario (Fig. 3c), when plants dispersed so rapidly that they were largely homogenized before insects had colonized the complete landscape. In contrast, the slowest loss of congruence was observed in the first scenario (Fig. 3a), in which both plants and insects colonized the landscape slowly.

\section{DISCUSSION}

\section{Shared large-scale spatial genetic structures in the beetle and its host plant}

Our study focused on unravelling the evolutionary processes taking place in a plant-insect interaction in which the plant disperses freely while the insect is constrained by its dependence on the host. Two outcomes were possible, considering either congruent or incongruent phylogeographic patterns between the plant and the insect. Our results clearly supported the former hypothesis, as global and significant overlap in the spatial genetic structures of both organisms was outlined by AFLPs (Fig. 1) and gene sequencing of mtDNA (Fig. 2; Appendix S3a, b). Phylogeographic structures, in contrast to diversity or adaptive patterns, can usually be satisfactorily detected using a limited number of specimens per population (e.g. Thiel-Egenter et al., 2011). Thus, our inferences are robust to the available sampling effort.

Divergence time estimates from mtDNA showed that the differentiation of beetle lineages originated before the Last Glacial Maximum (c. 18,000 years ago; COHMAP, 1998), during the middle of the Quaternary. The high congruence between mtDNA clades and AFLP clusters suggests that the former provides a reliable proxy for estimating divergence time among lineages. Assuming that the most recent common ancestor of all beetle lineages was monophagous (the most parsimonious expectation), our results suggest that the interaction between $O$. gloriosa and P. ostruthium has probably spanned several glacial cycles. Our results might reflect the specialized feeding behaviour of the beetle, which could have constrained its dispersal, at least during the early phases of landscape recolonization. This hypothesis is consistent with classical studies showing a narrower geographic range for herbivorous insects compared with that of their host plants (Strong, 1979), a trend that is even stronger in monophagous species (Quinn et al., 1998). Nevertheless, differences in dispersal abilities between the two species [i.e. the beetle shows few adaptations to dispersal (Kalberer et al., 2005) in contrast to the plant, which is wind-dispersed (Landolt et al., 2010)] should have decreased their global phylogeographic congruence, as theoretically expected (e.g. Nieberding \& Olivieri, 2007; Alvarez et al., 2010). Other processes, such as ongoing post-glacial recolonization, might thus be at work here. As shown by our application of a random-walk model, a pattern of phylogeographic congruence could be compatible with differences in dispersal abilities shortly after the recolonization process was completed (Fig. 3 and see below).

\section{Discrepancies in phylogeographies at a regional scale}

The high level of congruence recognized at a large spatial (and evolutionary) scale in the genetic structures of the plant and the beetle was not always maintained at a regional scale (Fig. 1). This was notably the case in the western and central Alps, with discrepancies corresponding to previously reported 
(a) slow plant and slow insect

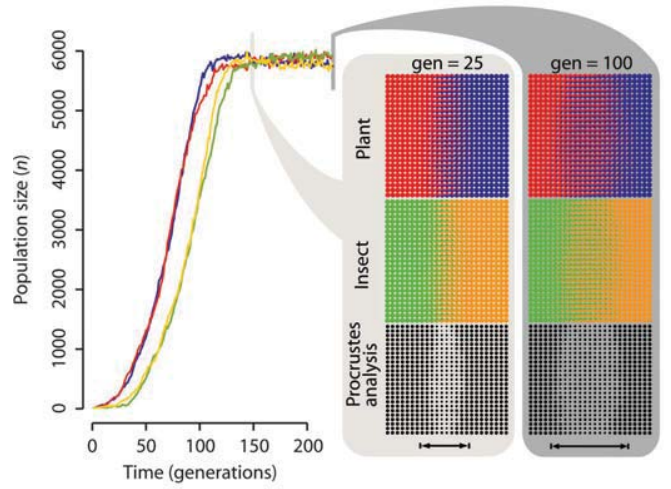

(c) rapid plant and slow insect

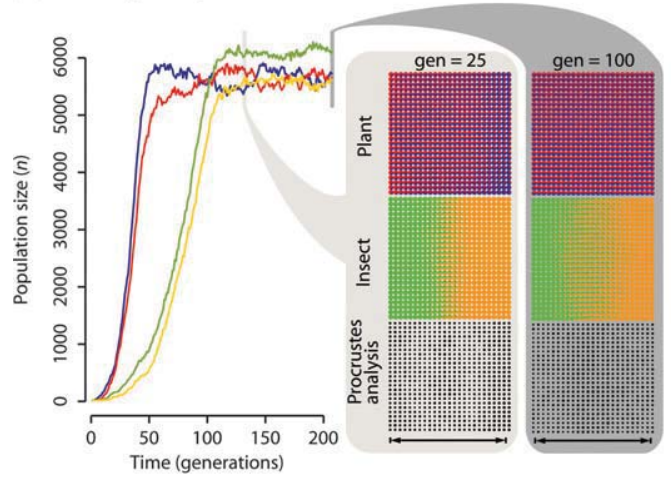

(b) slow plant and rapid insect

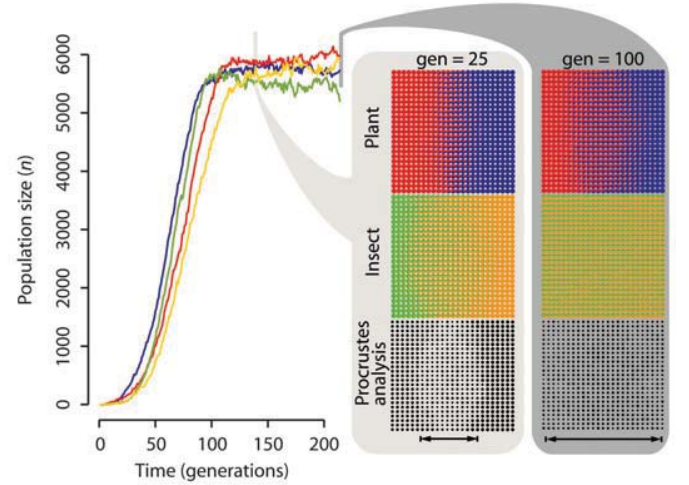

(d) rapid plant and rapid insect

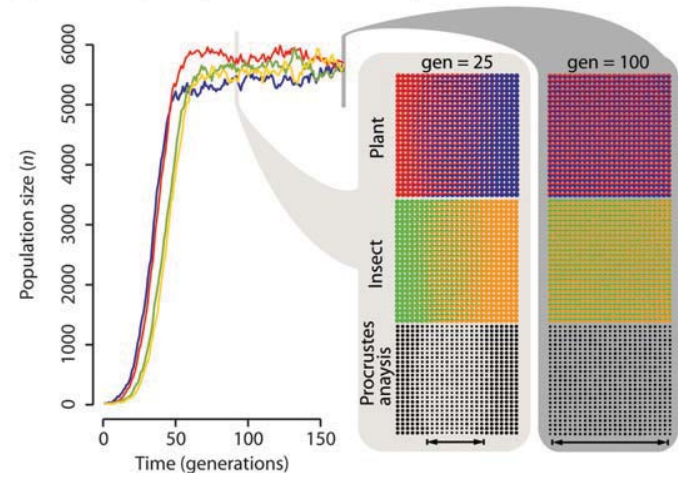

Figure 3 Results from the random-walk models simulating dispersal in a plant and its monophagous insect (see text for more details). The recolonization of an empty landscape (a 25 cell $\times 25$ cell grid) was simulated for two lineages of plants (in red and blue, respectively) and insects (in green and orange, respectively). Landscape recolonization started from 'refugial' cells at the left and right edges of the grid, with individuals dispersing over about 200 generations (dispersal parameters were obtained from random distributions; see text). Plants could establish in any grid cell while insects were restricted to cells where plants were already present. The number of plants and insects occupying the grid was measured during the course of the colonization process (left panel: population growth curves with one colour per lineage). The spatial distribution of plant and insect lineages (corresponding to phylogeographic patterns) were recorded at 25 and 100 generations after plants and insects had colonized $90 \%$ of the grid (centre and right panels, at gen $=25$ and gen $=100$, respectively). The respective phylogeographic patterns of plants and insects $(25$ cell $\times 25$ cell grids of populations represented as tiny pie-charts, with colours corresponding to lineages $)$ and their congruence quantified by the Procrustes analysis (i.e. circles are proportional to the plant-insect congruence; arrows correspond to suture zones) were displayed in lattices. Four scenarios were investigated by setting the respective dispersal speeds of plant and insects: (a) slow plants and slow insects, (b) slow plants and rapid insects, (c) rapid plants and slow insects, and (d) rapid plants and rapid insects.

phylogeographic suture zones (Fig. 1c; Schönswetter et al., 2005; Thiel-Egenter et al., 2011). For instance, the northern limits between the green, red and yellow clusters were not the same for plants and beetles, and the central Swiss Alps were dominated by the yellow group in both species but showed two additional spatially restricted lineages in the beetle (blue and black lineages; Fig 1a). In contrast to recent studies on hostparasite comparative phylogeography, in which the history of a parasite is considered as a proxy for that of the host (e.g. Nieberding \& Olivieri, 2007), the partial disconnection found here at a regional scale shows that the dependent and independent organisms could disperse using different pathways. It is therefore possible that different Alpine plant lineages escaped from the beetles during the Quaternary, which is consistent with the fact that $P$. ostruthium is present in other European mountain ranges that are not inhabited by the beetle (e.g. the Pyrenees, the Apennines and Corsica). Alternatively, stochastic processes could have resulted in regional discrepancies, as shown by our random-walk models (see below).

\section{Processes beyond the observed spatial patterns}

Using simulations, we showed that a random-walk recolonization of the landscape could produce phylogeographic patterns similar to those observed here (Fig. 3). Indeed, three scenarios result in large-scale phylogeographic congruence between the plant and the insect associated with regional discrepancies distributed along suture zones, where lineages originating from distinct refuges met: first, in recently colonized landscapes (i.e. when little time was given for homogenization after recolonization following the release of suitable habitats) with a 'rapid plant and rapid insect' scenario; second, also in recently 
colonized landscapes, with a 'slow plant and rapid insect' scenario; third, with a 'slow plant and slow insect' scenario irrespective of the timeframe. However, biological characteristics indicate that the plant disperses well (Landolt et al., 2010), whereas the beetle is a poorer disperser (Kalberer et al., 2005). Low dispersal abilities of the latter were confirmed based on genetic data in other Oreina species (Margraf et al., 2007; Triponez et al., 2011). As a consequence, we considered the 'rapid plant and slow insect' scenario, taken very shortly after recolonization was achieved (e.g. gen $=2$; data not shown), as the most realistic to explain our empirical results. Such a scenario would also fit well with the sequential evolution theory proposed by Jermy (1984), in which the insect progressively adapts to phenotypic changes in the plant, the latter not being influenced by the insect's evolutionary history.

\section{Inferences in a changing climate}

While the two species showed parallels in dispersal pathways at a large spatial scale, their respective fates under changing environmental conditions might differ, as suggested by the incongruent patterns in spatial genetic structures seen at regional scales. Because the dependent beetle species must spread independently from its host, this means that their relationship - and therefore the survival of the dependent species - is vulnerable: when dispersing into new areas, beetles are dependent on suitable microclimatic conditions, but also on a supplementary, unpredictable factor, i.e. finding a habitat harbouring the host. Stochastic parameters thus strongly reduce the rate of successful dispersal in the beetle, which would require a longer time period to disperse effectively in the pursuit of its ecological niche during climate shifts, such as the one we are currently experiencing. This conclusion strongly contrasts with the example of bruchid beetles: these speciesspecific phytophagous insects are specialized on angiosperm seeds and tend to disperse within the seed of their host (Alvarez et al., 2005, 2006, 2007), therefore increasing the chance of success of dispersal and establishment of new populations on their specific host plant. Because the dispersal of $O$. gloriosa does not closely follow the dispersal routes of its host plant at a regional scale, the interaction between these two organisms is potentially threatened during climatic oscillations that put pressure on alpine habitats. To what extent past abrupt Quaternary climate change events (Hewitt, 2000; Mayewski et al., 2004) have been associated with local extinctions, owing to co-evolutionary constraints, remains an open question.

\section{ACKNOWLEDGEMENTS}

We are grateful to the members of the IntraBioDiv Consortium (see Appendix S1 in Thiel-Egenter et al., 2011) for collecting and performing AFLP on the plant samples used in this study. The authors thank Felix Gugerli (Swiss Federal Research Institute WSL, Birmensdorf, Switzerland), Conrad Labandeira (Smithsonian Institution, Washington, DC, USA), two anonymous referees, and the editor, Melodie McGeoch, for useful comments on the manuscript. Matthias Borer thanks Makiala Kisanga for an introduction to the AFLP laboratory methods, and Ana Pinto, Franz Borer, Christoph Germann and Yann Triponez for help in collecting beetles. This research is part of the POMAPII project (Phylogeography of Mutualistic versus Antagonistic Plant-Insect Interactions), granted to Nadir Alvarez by the Swiss National Science Foundation (SNSF; grant 3100A0-116778). Matthias Borer was funded by a doctoral assistantship from the University of Neuchâtel. Nadir Alvarez and Nils Arrigo were funded by the SNSF (Ambizione fellowship PZ00P3_126624 and prospective researcher fellowship PBNEP-132747, respectively). Financial support to Sven Buerki was provided by Marie-Curie Intra-European Fellowship (CRADLE; no. 253866).

\section{REFERENCES}

Aeschimann, D., Lauber, K., Moser, D.M. \& Theurillat, J.-P. (2004) Flora Alpina. Haupt, Bern.

Alvarez, N., McKey, D., Hossaert-McKey, M., Born, C., Mercier, L. \& Benrey, B. (2005) Ancient and recent evolutionary history of the bruchid beetle, Acanthoscelides obtectus Say, a cosmopolitan pest of beans. Molecular Ecology, 14, 10151024.

Alvarez, N., Romero Napoles, J., Anton, K.-W., Benrey, B. \& Hossaert-McKey, M. (2006) Phylogenetic relationships in the Neotropical bruchid genus Acanthoscelides (Bruchinae, Bruchidae, Coleoptera). Journal of Zoological Systematics and Evolutionary Research, 44, 63-74.

Alvarez, N., Hossaert-McKey, M., Restoux, G., Delgado-Salinas, A. \& Benrey, B. (2007) Anthropogenic effects on population genetics of phytophagous insects associated with domesticated plants. Evolution, 61, 2986-2996.

Alvarez, N., Arrigo, N. \& IntraBioDiv Consortium (2008) SIMIL: an R (CRAN) scripts collection for computing genetic structure similarities based on STRUCTURE 2 outputs. Molecular Ecology Resources, 8, 757-762.

Alvarez, N., Thiel-Egenter, C., Tribsch, A. et al. (2009) History or ecology? Substrate type as a major driver of spatial genetic structure in Alpine plants. Ecology Letters, 12, 632-640.

Alvarez, N., Kjellberg, F., McKey, D. \& Hossaert-McKey, M. (2010) Phylogeography and historical biogeography of obligate specific mutualisms. The geography of host-parasite interactions (ed. by S. Morand and B. Krasnov), pp. 31-39. Oxford University Press, Oxford.

Arrigo, N., Tuszynski, J.W., Ehrich, D., Gerdes, T. \& Alvarez, N. (2009) Evaluating the impact of scoring parameters on the structure of intra-specific genetic variation using RawGeno, an $\mathrm{R}$ package for automating AFLP scoring. BMC Bioinformatics, 10, 33.

Bennett, K.D. (1990) Milankovitch cycles and their effects on species in ecological and evolutionary time. Paleobiology, 16, $11-21$.

Borer, M., Alvarez, N., Buerki, S., Margraf, N., Rahier, M. \& Naisbit, R.E. (2010) The phylogeography of an alpine leaf 
beetle: divergence within Oreina elongata spans the Quaternary. Molecular Phylogenetics and Evolution, 57, 703-709.

Bruyndonckx, N., Dubey, S., Ruedi, M. \& Christe, P. (2009) Molecular cophylogenetic relationships between European bats and their ectoparasitic mites (Acari, Spinturnicidae). Molecular Phylogenetics and Evolution, 51, 227-237.

Buerki, S., Forest, F., Acevedo-Rodríguez, P., Callmander, M.W., Nylander, J.A.A., Harrington, M., Sanmartín, I., Küpfer, P. \& Alvarez, N. (2009) Plastid and nuclear DNA markers reveal intricate relationships at subfamilial and tribal levels in the soapberry family (Sapindaceae). Molecular Phylogenetics and Evolution, 51, 238-258.

COHMAP (1988) Climatic changes of the last 18,000 years: observations and model simulations. Science, 241, 10431052.

Coope, G.R. (1990) The invasion of northern Europe during the Quaternary by Mediterranean species of Coleoptera. Biological invasions in Europe and the Mediterranean Basin (ed. by F. Di Castri, A.J. Hansen and M. Debussche), pp. 203-215. Kluwer, Dordrecht.

Dobler, S., Mardulyn, P., Pasteels, J.M. \& Rowell-Rahier, M. (1996) Host-plant switches and the evolution of chemical defense and life history in the leaf beetle genus Oreina. Evolution, 50, 2373-2386.

Drummond, A.J. \& Rambaut, A. (2007) BEAST: Bayesian evolutionary analysis by sampling trees. BMC Evolutionary Biology, 7, 214-221.

Emerson, B.C. \& Hewitt, G. (2005) Phylogeography. Current Biology, 15, R367-R371.

Espíndola, A. \& Alvarez, N. (2011) Comparative phylogeography in a specific and obligate pollination antagonism. PLOS ONE, 6, e28662.

Evanno, G., Regnaut, S. \& Goudet, J. (2005) Detecting the number of clusters of individuals using the software Structure: a simulation study. Molecular Ecology, 14, 2611-2620.

Falush, D., Stephens, M. \& Pritchard, J.K. (2007) Inference of population structure using multilocus genotype data: dominant markers and null alleles. Molecular Ecology Notes, 7, 574-578.

Gugerli, F., Englisch, T., Niklfeld, H., Tribsch, A., Mirek, Z., Ronikier, M., Zimmermann, N.E., Holderegger, R., Taberlet, P. \& IntraBioDiv Consortium (2008) Relationships among levels of biodiversity and the relevance of intraspecific diversity in conservation - a project synopsis. Perspectives in Plant Ecology, Evolution and Systematics, 10, 259-281.

Hadaček, F., Müller, C., Werner, A., Greger, H. \& Proksch, P. (1994) Analysis, isolation and insecticidal activity of linear furanocoumarins and other coumarin derivatives from Peucedanum (Apiaceae: Apioideae). Journal of Chemical Ecology, 20, 2035-2054.

Hall, T.A. (1999) BioEdit: a user-friendly biological sequence alignment editor and analysis program for Windows 95/98/ NT. Nucleic Acids Symposium Series, 41, 95-98.

Hayward, A. \& Stone, G.N. (2006) Comparative phylogeography across two trophic levels: the oak gall wasp Andricus kollari and its chalcid parasitoid Megastigmus stigmatizans. Molecular Ecology, 15, 479-489.

Hewitt, G.M. (1996) Some genetic consequences of ice ages, and their role in divergence and speciation. Biological Journal of the Linnean Society, 48, 247-276.

Hewitt, G.M. (2000) The genetic legacy of the Quaternary ice ages. Science, 405, 907-913.

Hsiao, T.H. \& Pasteels, J.M. (1999) Evolution of host-plant affiliation and chemical defense in Chrysolina-Oreina leaf beetles as revealed by mtDNA phylogenies. Advances in Chrysomelidae biology, Vol. 1 (ed. by M.L. Cox), pp. 321342. Backhuys Publishers, Leiden.

Jablonski, D. (2008) Biotic interactions and macroevolution: extensions and mismatches across scales and levels. Evolution, 62, 715-739.

Jablonski, D. \& Sepkoski, J.J. (1996) Paleobiology, community ecology, and scales of ecological pattern. Ecology, 77, 13671378.

Jackson, D.A. (1995) PROTEST: a Procrustean randomization test of community environment concordance. Ecoscience, 2, 297-303.

Jackson, S.T. \& Overpeck, J.T. (2000) Responses of plant populations and communities to environmental changes of the late Quaternary. Paleobiology, 26, 194-220.

Jermy, T. (1984) Evolution of insect/host plant relationships. The American Naturalist, 124, 609-630.

Kalberer, N.M., Turlings, T.C.J. \& Rahier, M. (2005) An alternative hibernation strategy involving sun-exposed 'hotspots', dispersal by flight, and host plant finding by olfaction in an alpine leaf beetle. Entomologia Experimentalis et Applicata, 114, 189-196.

Kendall, D.G. (1989) A survey of the statistical theory of shape. Statistical Science, 4, 87-99.

Kippenberg, H. (1994) Familie Chrysomelidae. Die Käfer Mitteleuropas, Vol. 3 (ed. by G.A. Lohse and W. Lucht), pp. 65-83. Goecke and Evers, Krefeld.

Klingenberg, C.P. \& McIntyre, G.S. (1998) Geometric morphometrics of developmental instability: analysing patterns of fluctuating asymmetry with Procrustes methods. Evolution, 52, 1353-1375.

Landolt, E., Bäumler, B., Erhardt, A., Hegg, O., Klötzli, F., Lämmler, W., Nobis, M., Rudmann-Maurer, K., Schweingruber, F., Theurillat, J.-P., Urmi, E., Vust, M. \& Wohlgemuth, T. (2010) Flora Indicativa. Ökologische Zeigerwerte und biologische Kennzeichen zur Flora der Schweiz und der Alpen. Haupt, Bern.

Larmuseau, M., Raeymaekers, J., Hellemans, B., Van Houdt, J. \& Volckaert, F. (2010) Mito-nuclear discordance in the degree of population differentiation in a marine goby. Heredity, 105, 532-542.

Legendre, P. \& Lapointe, F.J. (2004) Assessing the congruence among distance matrices: single malt Scotch whiskies revisited. Australian and New Zealand Journal of Statistics, 46, 615-629.

Mardulyn, P., Milinkovitch, M.C. \& Pasteels, J.M. (1997) Phylogenetic analyses of DNA and allozyme data suggest 
that Gonioctena leaf beetles (Coleoptera; Chrysomelidae) experienced convergent evolution in their history of hostplant family shifts. Systematic Biology, 46, 722-747.

Margraf, N., Verdon, A., Rahier, M. \& Naisbit, R.E. (2007) Glacial survival and local adaptation in an alpine leaf beetle. Molecular Ecology, 16, 2333-2343.

Mayewski, P.A., Rohling, E.E., Stager, J.C., Karlen, W., Maasch, K.A., Meeker, L.D., Meyerson, E.A., Gasse, F., van Kreveld, S., Holmgren, K., Lee-Thorp, J., Rosqvist, G., Rack, F., Staubwasser, M., Schneider, R.R. \& Steig, E.J. (2004) Holocene climate variability. Quaternary Research, 62, 243-255.

Mouritsen, K.N. \& Poulin, R. (2002) Parasitism, climate oscillations and the structure of natural communities. Oikos, 97, 462-468.

Nieberding, C. \& Olivieri, I. (2007) Parasites: proxies for host history and ecology? Trends in Ecology and Evolution, 22, 156-65.

Nylander, J.A.A. (2004) MrModeltest v2. Program distributed by the author. Evolutionary Biology Centre, Uppsala University, Uppsala. Available at: http://www.abc.se/ nylander/ mrmodeltest $2 /$ mrmodeltest $2 . h t m l$.

Peres-Neto, P.R. \& Jackson, D.A. (2001) How well do multivariate data sets match? The robustness and flexibility of a Procrustean superimposition approach over the Mantel test. Oecologia, 129, 169-178.

Quinn, R.M., Gaston, K.J. \& Roy, D.B. (1998) Coincidence in the distribution of butterflies and their food plants. Ecography, 21, 279-288.

Rambaut, A. \& Drummond, A.J. (2007) Tracer v1.5. Program distributed by the authors. Institute of Evolutionary Biology, University of Edinburgh, Edinburgh. Available at: http:// tree.bio.ed.ac.uk/software/tracer.

Rasmann, S., Alvarez, N. \& Pellissier, L. (in press) The altitudinal niche breadth hypothesis in plant-insect interaction. Plant-insect interactions (ed. by C. Voelckel and G. Jander). Annual Plant Reviews Series. Wiley-Blackwell Publishing, Oxford.

Schmitt, T. (2007) Molecular biogeography of Europe: Quaternary cycles and postglacial trends. Frontiers in Zoology, 4, 11.

Schönswetter, P., Stehlik, I., Holderegger, R. \& Tribsch, A. (2005) Molecular evidence for glacial refugia of mountain plants in the European Alps. Molecular Ecology, 14, 3547-3555.

Simon, C., Frati, F., Beckenbach, A., Crespi, B., Liu, H. \& Flook, P. (1994) Evolution, weighting, and phylogenetic utility of mitochondrial gene-sequences and a compilation of conserved polymerase chain reaction primers. Annals of the Entomological Society of America, 87, 651-701.

Sorenson, M.D. \& Franzosa, E.A. (2007) TreeRot. v3. Program distributed by the authors. Department of Biology, Boston University, Boston, MA. Available at: http://people.bu.edu/ msoren/TreeRot.html.

Stewart, J.R., Lister, A.M., Barnes, I. \& Dalén, L. (2010) Refugia revisited: individualistic responses of species in space and time. Proceedings of the Royal Society B: Biological Sciences, 277, 661-671.
Strong, D. (1979) Biogeographic dynamics of insect-host plant communities. Annual Review of Entomology, 24, 89-119.

Swofford, D.L. (2003) PAUP*. Phylogenetic analysis using parsimony (and other methods), version 4.0b10. Sinauer Associates, Sunderland, MA.

Taberlet, P., Fumagalli, L., Wust-Saucy, A.-G. \& Cosson, J.F. (1998) Comparative phylogeography and postglacial colonization routes in Europe. Molecular Ecology, 7, 453-464.

Tamura, K., Dudley, J., Nei, M. \& Kumar, S. (2007) MEGA4: molecular evolutionary genetics analysis (MEGA) software version 4.0. Molecular Biology and Evolution, 24, 15961599.

Thiel-Egenter, C., Gugerli, F., Alvarez, N. et al. (2009) Effects of life history traits on the genetic diversity of high-mountain plants: a multi-species study across the Alps and the Carpathians. Global Ecology and Biogeography, 18, 78-87.

Thiel-Egenter, C., Alvarez, N., Holderegger, R. et al. (2011) Break zones in the distributions of alleles and species in alpine plants. Journal of Biogeography, 38, 772-782.

Thioulouse, J., Chessel, D., Dolédec, S. \& Olivier, J.M. (1996) ADE-4: a multivariate analysis and graphical display software. Statistics and Computing, 7, 75-83.

Thompson, J.D., Higgins, D.G. \& Gibson, T.J. (1994) CLUSTAL W: improving the sensitivity of progressive multiple sequence alignment through sequence weighting, positionspecific gap penalties and weight matrix choice. Nucleic Acids Research, 22, 4673-4680.

Triponez, Y., Buerki, S., Borer, M., Naisbit, R.E., Rahier, M. \& Alvarez, N. (2011) Discordances between phylogenetic and morphological patterns in alpine leaf beetles attest to an intricate biogeographic history of lineages in postglacial Europe. Molecular Ecology, 20, 2442-2463.

Vos, P., Hogers, R., Bleeker, M., Reijans, M., Vandelee, T., Hornes, M., Frijters, A., Pot, J., Peleman, J., Kuiper, M. \& Zabeau, M. (1995) AFLP: a new technique for DNA fingerprinting. Nucleic Acids Research, 23, 4407-4414.

Willis, K.J. \& van Andel, T.H. (2004) Trees or no trees? The environments of central and eastern Europe during the Last Glaciation. Quaternary Science Reviews, 23, 2369-2387.

\section{SUPPORTING INFORMATION}

Additional Supporting Information may be found in the online version of this article:

Appendix S1 Sampled populations of Oreina gloriosa and Peucedanum ostruthium with their geographical coordinates and the number of analysed individuals.

Appendix S2 Supplementary experimental procedures: crosscheck of the Procrustes results with a non-model based approach.

Appendix S3 Supplementary results: (a) strict consensus tree of the maximum parsimony analysis for the Oreina gloriosa sequence dataset; (b) contingency table comparing the grouping of O. gloriosa individuals using AFLP and mitochondrial DNA data. 